\title{
Baroque
}

\section{Communication de Jean-Claude Martinon}

Jean-Claude Martinon

\section{(2) OpenEdition}

\section{Journals}

Édition électronique

URL : http://journals.openedition.org/baroque/513

DOI : 10.4000/baroque.513

ISSN : 2261-639X

\section{Éditeur :}

Centre de recherches historiques - EHESS, Éditions Cocagne

\section{Édition imprimée}

Date de publication : 1 mai 1980

ISSN : 0067-4222

\section{Référence électronique}

Jean-Claude Martinon, «Communication de Jean-Claude Martinon », Baroque [En ligne], 09-10 | 1980, mis en ligne le 15 mai 2013, consulté le 30 avril 2019. URL : http://journals.openedition.org/ baroque/513 ; DOI : 10.4000/baroque.513

Ce document a été généré automatiquement le 30 avril 2019

(c) Tous droits réservés 


\title{
Communication de Jean-Claude Martinon
}

\author{
Jean-Claude Martinon
}

\section{Intervention}

1 Le titre même de notre colloque : "Statut du concept de baroque » nous questionne d'une manière qui n'est pas seulement méthodologique, mais qui est aussi épistémologique. J'y reviendrai certainement durant ces journées. Nous n'arriverons peut-être pas à clarifier le concept de baroque, mais ces journées auront le mérite de lever quelques ambiguïtés. Comment découper notre objet d'étude ? Le mot baroque n'est-il pas une manière de dire et de nommer des études bien différentes les unes des autres, ne s'agit-il pas d'une commodité du langage usuel afin de masquer la complexité des tâches et la dissémination du champ des études historiques. Aurions-nous affaire à un rituel du langage dont la fonction serait de baliser et de borner un espace de communication? Même si les spécialistes ne parlent pas de la même chose, un consensus flou serait pré-construit et agirait comme lieu de reconnaissance plus que comme méthode de connaissance. Chacun pourrait ainsi reconnaître un terrain d'entente avec d'autres spécialistes, même si l'on ne parle pas exactement de la même chose. La méconnaissance implicite du statut du concept de «baroque » fonctionnerait alors comme une facilitation des rencontres entre spécialistes qui - laissant au vestiaire les couteaux (les concepts aux arêtes dures et tranchantes dont parle Nietzsche) de la spécialisation - pourraient, en terrain neutre et pourtant défini, parler ensemble d'événements, de discours, de formes esthétiques, de musiques, de fêtes, de tout un ensemble de faits, de systèmes et peut-être de structures faisant partie du baroque. La reconnaissance globale du phénomène baroque serait ainsi construit sur la méconnaissance du concept; l'entente plus ou moins floue de la définition et la détermination approximative du champ seraient les fondements de la communication. Le baroque - en deçà de la construction "patiente » du concept - serait alors un lieu de reconnaissance et serait accepté comme une matière soumise à un mouvement centrifuge qui, malgré l'opacité de s'a composition, aurait pour intérêt de 
nous réunir en deçà, et peut-être au-delà de nos territoires scientifiques propres. Peutêtre, après cette présentation au pessimisme tempéré, pourrions-nous penser le baroque comme un phénomène social total. Marcel Mauss, dès 1901 dans la Grande Encyclopédie, expliquait que «quelle que soit la grandeur et la forme (des) groupes et de ceux qu'on pourrait énumérer - classe, tribu, groupe professionnel, caste, commune - ils présentent tous ce caractère qu'ils sont formés par une pluralité de consciences individuelles, agissant et réagissant les unes les autres. C'est à l'épreuve de ces actions et de ces réactions, de ces interactions, que l'on reconnaît les sociétés : or la question est de savoir si, parmi les faits qui se passent au sein des groupes, il en est qui manifestent la nature du groupe en tant que groupe et non pas la nature des individus qui les composent, les attributs généraux de l'humanité ». Parler du phénomène baroque comme un fait social total, c'est essayer de découvrir les lois qui pourraient expliquer, à travers l'Europe, une mentalité baroque s'épanouissant aussi bien dans les arts, les lettres que dans les sciences et la théologie. C'est considérer le phénomène baroque comme spécifique et irréductible à d'autres phénomènes sociaux. C'est aussi, en deçà des spécialités. essayer de fonder, d'une manière non tautologique, un habitus baroque que l'on pourrait retrouver, malgré les variations, en Italie, en Allemagne, en Espagne, en Bohême et dans des régions françaises, l'Occitanie peut-être.

2 J'aurais aimé présenter ici un exemple qui pourrait devenir un paradigme et qui montre bien, du moins je le suppose, le statut interdisciplinaire du phénomène baroque ainsi que son extension. La complexité de la compréhension d'un exemple, fût-il ponctuel, suppose d'approcher le baroque comme un fait social total.

3 Mais ne soyons pas trop abstrait: prenons l'exemple que je voile de théorie depuis un moment. Il s'agit donc de deux types d'approches: la première est l'explication de la relation existant entre l'environnement social survenant en Bohême, au crépuscule de la défaite de la Montagne Blanche et les différents acteurs sociaux qui nous intéressent ici : le commanditaire, les architectes, le sculpteur. Nous en revenons donc à la question : qui commandite quoi à qui ? Le commanditaire : le Comte Sporck; l'objet : le palais, l'église et l'hôpital de Kuks; le sculpteur : Mathyas Braun ; les architectes : les frères Montalegre. 


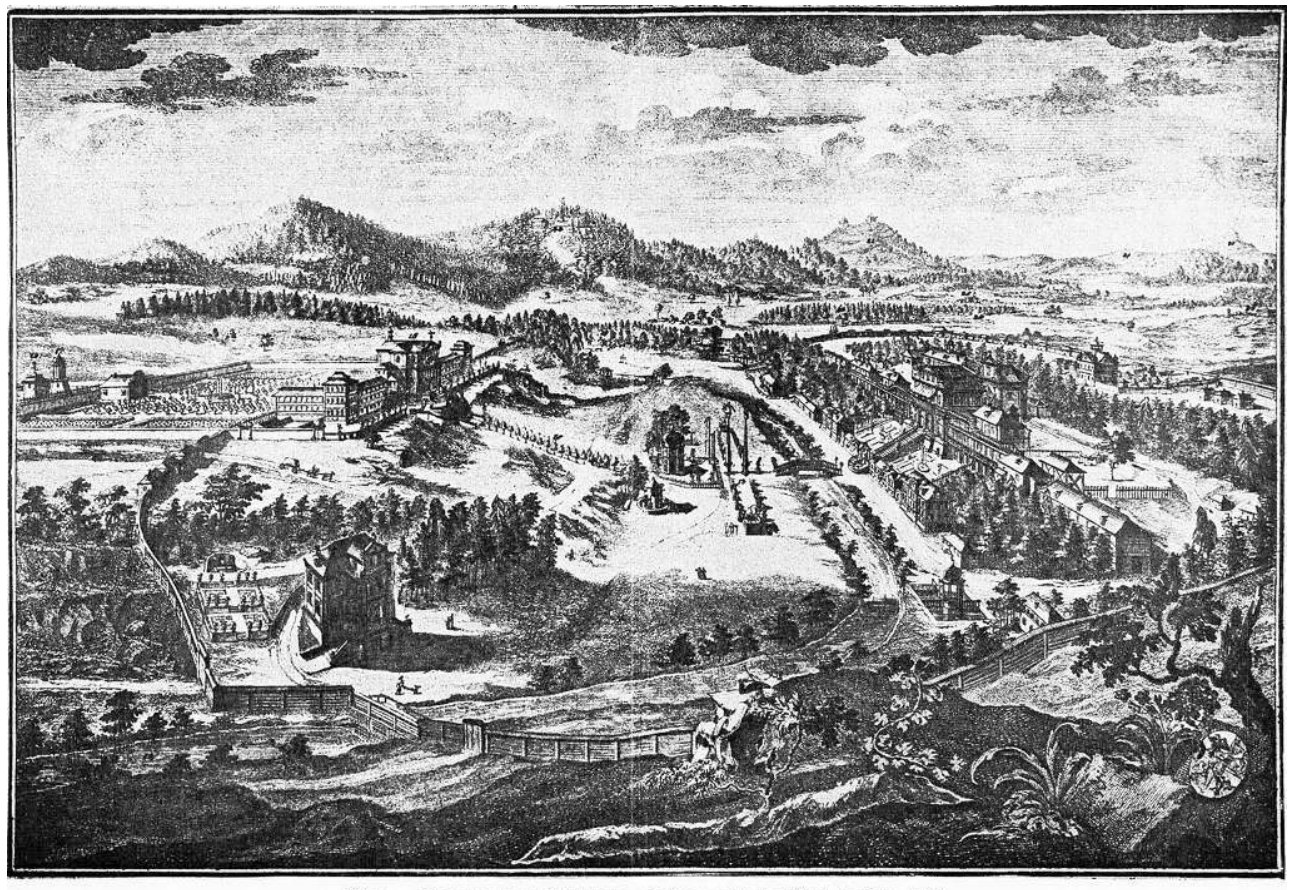

№ 1. - Michael et J. de Montalegre : Château, pont et hôpital de Kuks, 1742

Le commanditaire ne semble pas l'homme qui pourrait incarner le portrait idéal typique de cette société praguoise, celle des Kinsky, des Bucquoy, des Lobkowitz ${ }^{1}$. Sporck est fils d'un combattant de la "montagne blanche", mais d'un combattant roturier d'une sorte de Simplicius qui aurait eu de la chance et de la suite dans les idées: Sporck fils de reître est comte grâce à la rapière paternelle. Il détient la puissance, l'argent, mais il est différent de la noblesse qui, à ce moment-là possède la puissance et le prestige à Prague. On pourrait dire qu'il est marginal, comme Trimalcion « Venu d'Asie alors qu'il n'était pas plus haut qu'un candélabre » et qui d'esclave d'origine asiatique ou bien d'enfant trouvé sur un tas de fumier sera acheté par un grand seigneur puis affranchi et continuateur de la fonction et du nom de son maître après la mort de celui-ci. Trimalcion ne sera jamais un notable, il vivra sur un pied seigneurial, mais jamais il ne pourra entrer dans la noblesse équestre ou même municipale ${ }^{2}$. Sporck détient la puissance, il n'est pas légitime. En effet il est celui qui, grâce à la crise politique du début du XVII siècle grâce à la Défenestration de Prague, grâce à la Paix de Westphalie, c'est-à-dire grâce à l'exil d'une partie de la noblesse et de l'élite intellectuelle protestante, possède un NOM et un titre gagné par son père, possède aussi de grands domaines d'exploitation agricole qui durant le TEMNO - c'est-à-dire la période la plus sombre durant laquelle la Bohême est asservie à une aristocratie étrangère - ont changé de main et ont été reconstitués par de nouveaux propriétaires. Le Comte Sporck est donc un produit mixte, Comte grâce à l'empereur, propriétaire terrien, riche et puissant, mais aussi, en marge du prestige social détenu par les maîtres du nouveau quartier de Prague où s'édifient les palais: la Mala Strana. Rapidement brossé, voici le portrait ambigu du commanditaire; et vous voyez déjà la complexité de l'interprétation qu'on va pouvoir donner de cet Hôpital de Kuks - le palais ayant disparu - puisqu'il est commandé par un acteur - homme baroque s'il en est - situé en marge du groupe social dont il fait pourtant partie économiquement.

4 Le Comte Sporck confie aux frères Montalegre le soin de construire Kuks, mais, ce qui est important est surtout le fait suivant : il demande à Mathyas Braun de réaliser la série des 
statues des Vices et des Vertus ornant - si j'ose dire - la terrasse de l'Hôpital ainsi que de sculpter les rochers de la forêt de Bethléem, toute proche de Kuks.

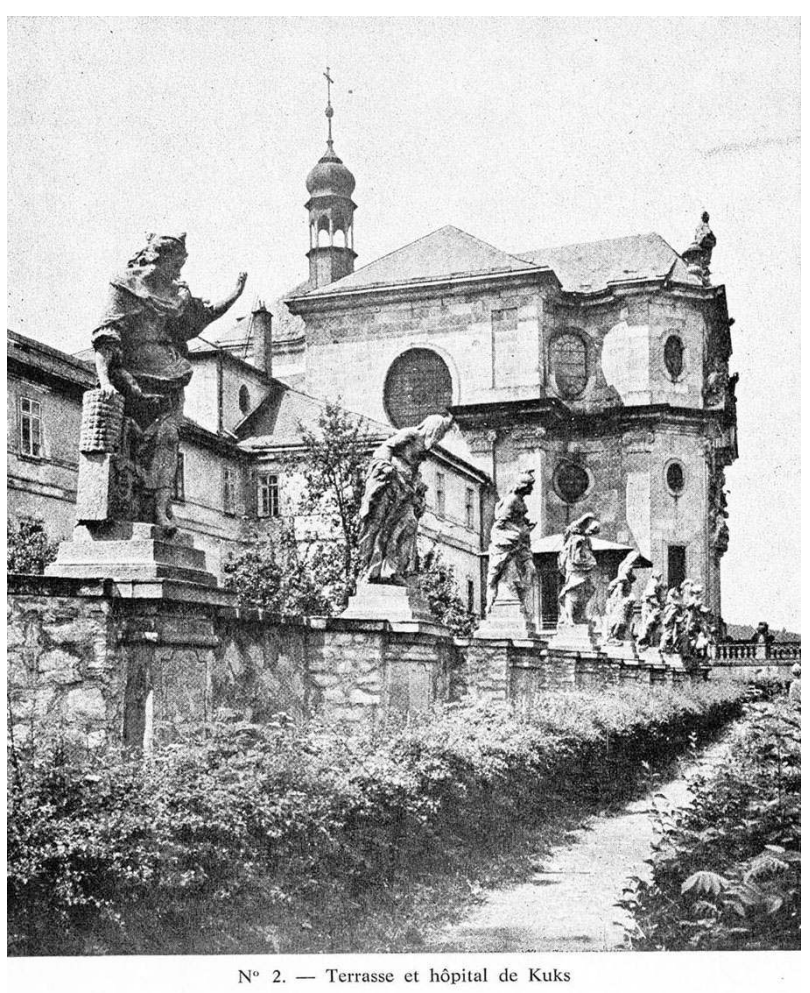

Mathyas Braun est Tyrolien mais toute sa production artistique se situe en Bohême. Les historiens de l'art, par exemple Bachmann, décrit les statues comme étant d'un style expressif et extatique, pleines de « fougue dramatique ». Braun est né en 1684 dans l'Oetzl en Tyrol, il fut appelé à Prague à l'âge de vingt-six ans par le comte Sporck. C'est à partir de 1719 qu'il sculpta les statues de Kuks. 


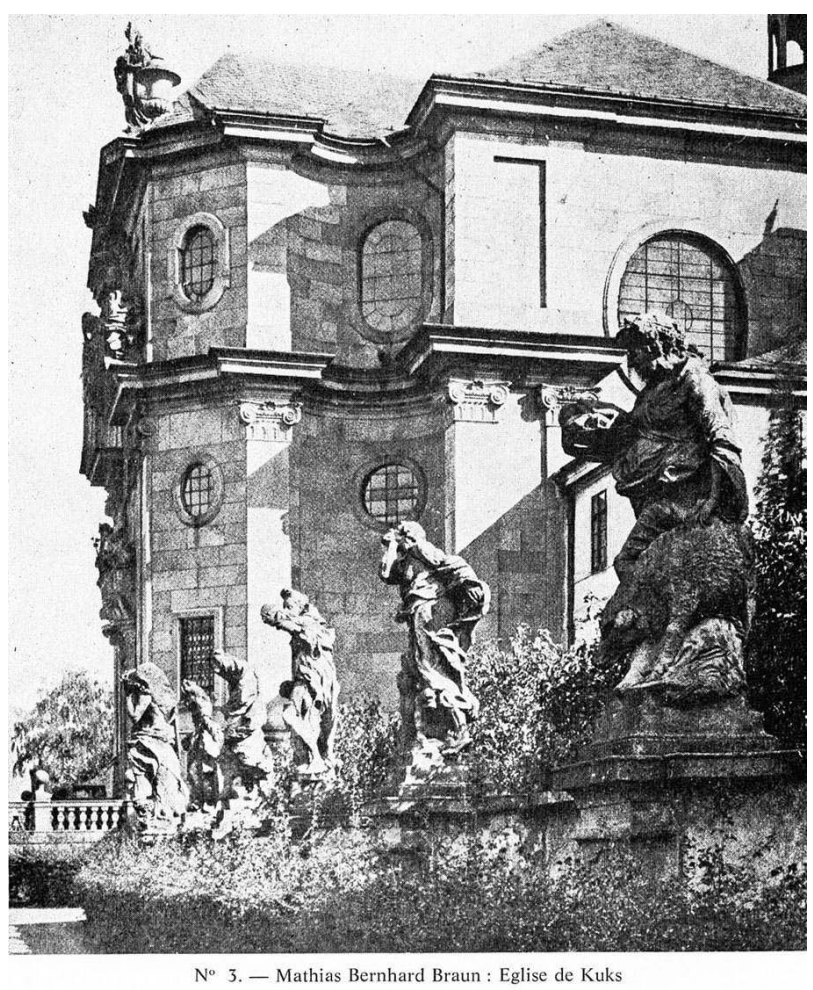

Il y aurait beaucoup à dire sur la vie de Mathyas Braun, il faudrait en faire la monographie, expliquer l'influence de Michel-Ange et du Bernin sur ce sculpteur tyrolien, il faudrait aussi se poser la question de savoir si ce concept d'influence est opératoire, c'est-à-dire s'il peut nous aider à comprendre, à classer ces archives de pierres que sont les statues. Il faudrait aussi s'intéresser, à la structure familiale des artistes: celle de Mathyas Braun est exemplaire si l'on veut comprendre ce qu'est un atelier, ce qu'est le passage de l'artisan à l'artiste dans la société moderne occidentale. Il faudrait alors décrire l'atelier de Mathyas Braun à Prague; la reprise de l'atelier du grand-oncle par Braun; sa position de patron légitime de l'atelier, faisant travailler son frère Dominique, son neveu Anton. Mais ce n'est pas le lieu ici d'expliquer la structuration historique d'un atelier, la position stratégique qu'il détient (la concurrence avec Brokoff par exemple et l'ampleur des commandes), bien que cette démarche autant d'historien que de sociologue soit fondamentale pour expliquer ce qu'a pu être le produit d'un tel faisceau social de relations et de concurrence.

Études sociales de la situation stratégique ambiguë du commanditaire; monographie de l'artiste et de son atelier, certes sont les premiers pas d'une analyse de sociologie historique. Elles nous font comprendre, non pas seulement le micro-milieu culturel permettant de produire telle ou telle œuvre mais elles peuvent être aussi une des clefs d'interprétation de l'œuvre elle-même, de sa situation historique et de son iconographie symbolique. Mais, deuxièmement, ce que je voudrais indiquer ici est une autre orientation de recherches qui n'a de sens que si les «familles » et si les archives de cette archéologie dont je viens de parler sont déjà précisées et analysées. Il s'agit de l'espace lui-même : que peut dire le sociologue de la construction de l'espace par Mathyas Braun, et par les frères Montalegre sur la demande du Comte Sporck?

6 Au premier abord, il s'agit du partage du monde. Topographie légendaire, topographie sociale, situation géographique exposent, à Kuks, la même règle, celle de la hiérarchie des 
ordres. Un vallon, deux pentes; d'un côté le palais barrant l'horizon de la colline, fastueux, le comte Sporck domine ses terres, mesure l'espace de la Bohême, le regard peut embrasser l'étendue de sa puissance. Il peut regarder et se montrer, mais il n'a qu'à se retourner pour être en présence du spectacle de son destin. De l'autre côté du vallon, sur la pente opposée, sont situés l'hôpital et l'église de Kuks; séparant le palais de l'église, un petit ruisseau serpente qu'un pont traverse. Pour descendre du palais, un escalier monumental relie directement le palais au pont. Pour accéder, de l'autre côté, à l'église, à l'hôpital, un escalier complexe, une terrasse théâtrale. Face à face, d'un côté les ors, les prestiges et les richesses, la puissance et l'orgueil, de l'autre la charité divine : l'ordre de l'empire terrestre se confronte à la puissance céleste. Le plan est presque symétrique entre ciel et terre : le pont étroit enjambe la rivière. On ne sait trop alors si j'expose le paradigme des relations sociales ou bien si je décris simplement l'espace baroque ainsi structuré. Il est possible à tout le moins de parler d'analogie, il est peut-être plus périlleux, sans études précises, de parler d'homologie structurale entre la position spatiale des objets architecturaux telle qu'elle se présente à Kuks et la vision du monde - laïque et religieuse - du groupe social dont faisait partie le comte Sporck. Mais c'est dans ce sens-là qu'une étude sociologique des rapports entre l'espace et la vision du monde d'un groupe, incarné par la création architecturale doit se développer : je parle surtout, ici, d'architecture, mais rentrons dans le détail des sculptures de Mathyas Braun sur la terrasse de l'hôpital et de l'église de Kuks. Kuks, c'est le théâtre : c'est une composition de l'espace tel qu'il ressemble à une scène, c'est un scénario construit en fonction de la présentation : les acteurs étant les statues de la terrasse, la pièce de théâtre jouée reste à déterminer et à mon sens l'iconographie la dévoile. Or, cette scène, cette terrasse n'est compréhensible que par la trajectoire que le spectateur doit suivre pour arriver au centre de la scène, au milieu des statues. C'est pourquoi la topographie des lieux a une grande importance. Partant de la rivière traversée sur un pont, il faut s'avancer sur un chemin qui s'arrête au pied de la colline et de là, pour accéder à la terrasse, il faut emprunter un escalier. Pour découvrir de plus près les statues penchées en avant, pour comprendre le sens de cette "geste de la pierre torturée", il faut emprunter l'escalier qui dans l'économie du site et du spectacle a une importance primordiale. "L'escalier n'est plus fait pour être contemplé mais pour être vécu » nous dit Charpentrat, et cette vie qui saisit l'homme entreprenant l'ascension vers la terrasse, c'est-à-dire vers l'hôpital et l'église, est par deux fois orienté vers des images et des objets de réflexions dont la mort (laquelle?) n'est pas absente: ici la mort s'organise elle-même en décor. Après la première volée de l'escalier, celui-ci se sépare laissant en son milieu une porte surmontée d'une tête de mort, bouche pouvant happer le spectateur, puis au niveau supérieur «L'ange de la mort bienheureuse (ou radieuse) » attend le spectateur.

7 Donc à trois niveaux, il faut découvrir la cohérence de la structure de l'œuvre architecturale baroque. C'est un spectacle, cela n'est pas nouveau, mais ici, il s'agit d'un spectacle en plein air. Il ne s'agit pas d'un décor intérieur comme à la Wies bavaroise ou à Saint Nicolas de Mala Strana. Mathyas Braun doit être considéré comme un sculpteur en plein air. À Kuks, le théâtre est une rencontre avec la puissance des images et malgré la danse allègre $d u \mathrm{XVIII}^{\mathrm{e}}$ siècle sur son volcan, il semble qu'une sorte de séduction des insignes mortels n'est pas absente de cette révérence des acteurs de la terrasse à la fin de la pièce. S'agirait-il alors d'une réflexion s'épanouissant entre le crâne et le masque? Ainsi il faut essayer de comprendre :

a - la topographie des lieux; 
b-le mouvement, «la trajectoire» que le spectateur est forcé de prendre de par la composition de l'espace ;

c-le sens de ce mouvement qui se résume dans sa conclusion : c'est-à-dire l'accès à la terrasse, c'est-à-dire encore le discours qui lui est tenu par les statues : il s'agit là d'une étude iconographique. Espace, cheminement et code, voici donc quelques variables déterminant la cohérence de l'œuvre.

8 À Kuks, les vices et les vertus sont personnifiées et les différentes sculptures n'ont pas seulement comme fonction d'orner la terrasse de l'hôpital et de l'église, mais elles nous proposent aussi une lecture, un texte dont elles sont les éléments syntaxiques. En effet, cette suite de statues est une introduction majeure aux grands thèmes iconographiques de la Bohême du début du XVIII ${ }^{e}$ siècle. Ces statues sont donc à la fois texte à déchiffrer, nous faisant comprendre la culture de l'époque, mais aussi œuvre d'art et mise en scène. Elles sont donc à analyser sur trois plans différents. Mots d'un texte, discours plastique sur une morale - celle de la régulation des passions -; ornement et masque puisque ce que ces statues nous disent dans la geste de pierre inscrite dans un cadre, elles nous le content au sein d'une page structurée avec ses marges et ses interlignes, ses blancs et ses noirs, avec sa structure en un mot, qui est ici l'architecture de Kuks, mais ce sont enfin des œuvres d'art et non pas seulement ce que Panofsky appelle un document. Certes, nous cherchons des documents à leur propos. Certes ces statues sont aussi documents dans la mesure où elles nous renseignent sur l'état des signes dans une culture donnée, mais elles sont aussi œuvre, statues en grès de Bohême dépassant leur statut de document pour devenir dans l'ironie qui préside à leurs mouvements, dans le jeu que les actrices peuvent interpréter au haut de la terrasse, œuvres d'art multipliant les sens et faisant de notre propre lecture, un siècle et demi plus tard, l'équivalent de mots croisés par lesquels nous pouvons interpréter l'interprétation de ces représentations. C'est pourquoi la complexité est extrême lorsqu'on aborde cette terrasse puisque le propre de celle-ci est justement dans la clarté d'une lecture bien menée de nous faire déceler la faille qui fait échapper au déterminisme d'un code ces purs fragments de l'apparence dite. Cet exemple, si l'on pouvait exhaustivement le présenter n'est pas une description architectonique, ni une analyse théologique ou bien encore une description ethnographique. Tout élément susceptible d'aider à la compréhension du phénomène devrait non pas s'ajouter mais bien entrer en interaction avec les autres éléments, et ceci à plusieurs niveaux d'explications. C'est la somme des interactions formant peut-être, si nous sommes optimistes, systèmes, signifie comme total, un fait social à la fois économique, social, théologique et esthétique. Étudier un petit exemple, c'est avoir à l'esprit la totalité des interactions qui le composent, c'est peut-être déjà, opératoirement, savoir - et non pas seulement croire que le concept de baroque existe.

\section{Discussion}

- KRYNEN : L'interdisciplinarité ne sera jamais qu'une connivence entre spécialistes. Nous n'aurons pas la prétention d'aboutir à des schémas méthodologiques qu'on n'aurait plus qu'à appliquer aux diverses spécialités.

10 - COURTÈS : Oui. Le Baroque va au devant de l'interdisciplinarité. Il y a moins une essence du Baroque qu'une histoire de son expansion. 
11 - BRUNON : Je relève l'importance du nombre 2 dans le rapport de l'un et du multiple. Tartuffe se définit dans une dualité : ce qui se passe au-dessus et au-dessous de la table... Nulle rupture entre les deux plans. Le vrai Tartuffe est-il seulement ce jeu de jambes et de pulsions au-dessous de la table? N'est-il pas précisément contradiction des deux plans?

12 - KRYNEN : La duplicité est plus caractéristique du Baroque que la dualité. Répulsion des contraires. La nature et la grâce. Affrontement. Accord impossible.

13 -COURTÈS: La notion d'impossible m'intéresse, laquelle tôt ou tard aboutit à l'éclatement. Quant au nombre 2, c'est la duplicité en effet, et le dramatique.

14 - AUZIAS : Faut-il faire constamment référence à la théologie ? Un monde où tant de gens sont malades ou marginaux s'inscrit d'autre part contre cette suprématie du code dont on a parlé.

15 - COURTÈS : Jean Hippolyte remarque, dans un texte sur Hegel : le langage dit tout, il dit même qu'il a des limites. Il en va de même de la théologie : elle dit aussi que l'homme est perdu. S'il y a un désespoir, il peut se formuler théologiquement.

16 - Suit un échange de vues sur les tabous et sur le vécu populaire, que certaines œuvres traduisent, y compris des œuvres d'origine savante ou des sermons, à résonance baroque.

17 - CASTAN : L'essentiel de l'intervention de M. Courtès tient, me semble-t-il, dans une critique fondamentale de tout formalisme et de toute scholastique dans l'appréhension du phénomène de civilisation. La propension naturelle est de catégoriser les conclusions que l'on tire. Et donc cette critique, à elle seule, justifierait notre réunion: critique de l'intérieur. Ce qui fait l'unité des démarches dans les divers secteurs de la recherche. C'est moins un concept que la manière de procéder dans la connaissance. L'échange collectif concernant les méthodologies est de nature à harmoniser les points de vue et à fonder la cohérence d'une science du Baroque.

18 - MARTINON : M. Courtès, pensez-vous donc à une affinité entre la situation du chercheur et le lieu même de la recherche? Après trois ou quatre siècles, nous ne sommes plus dans la même situation que l'homme de ce moment historique. Comment mettre en œuvre cette idée?

19 - COURTÈS : Qu'est-ce qui est premier dans le chercheur? N'est-ce pas son intelligence? La façon dont le Baroque fonctionne est homologue de la façon dont fonctionne l'intelligence du chercheur.

20 - WAGNER : La qualité primordiale du chercheur, c'est l'enthousiasme.

21 - COURTÈS : Non point la patience, mais la passion!

22 - MARTINON : Comment différencier cette passion qui serait celle d'un artiste en affinité avec le lieu du Baroque du désir de rectitude proprement scientifique?

23 - COURTÈS : La différence se fait dans l'expression et dans le point d'aboutissement. D'autre part, y a-t-il un poète aujourd'hui qui ne soit un chercheur en poésie et qui n'ait parallèlement à sa création poétique quelque chose à dire sur le langage ? Je pensais à cela après lecture d'un article sur Audiberti paru dans Baroque en voyant La Logeuse de cet auteur (un personnage traversé, agi par le langage), et en lisant L'ouvre-boîte, essai par le même auteur, d'interprétation de l'art moderne. Quand il théorise, il se distingue du chercheur en titre, qui doit avoir l'air ennuyeux pour authentifier la qualité de ses recherches. 
24 - WAGNER: À quel moment situez-vous l'intervention du concept, dans le domaine philosophique et scientifique? À l'époque que nous considérons on s'est peu occupé de conceptualisation. Phénomène récent, qui est en train d'être évacué, par les scientifiques eux-mêmes.

25 - COURTÈs: Où donc est le concept chez Fermat, chez Maupertuis? On a oublié d'enseigner dans les manuels de philosophie, qu'il y avait une coupure radicale entre un certain discours scientifique et l'intuition.

26 - WAGNER : C'est à la fin du XIX ${ }^{\mathrm{e}}$ siècle que la notion de concept a conquis abusivement son importance.

27 - COURTÈS : Il y a eu contamination de l'axiomatique, qui est tout autre chose.

28 - BRUNON : Si nous renonçons au concept, ne faut-il pas étudier des fonctionnements?

29 - COSTABEL : Je penserais aux classements, aux passages, aux paliers établis selon les niveaux entre des domaines différents... Vous nous avez mis en garde contre la conceptualisation. Il faudrait maintenant s'entendre sur la périodisation.

30 - AUZIAS: Au théâtre, des formes peuvent être récurrentes avec des contenus sociologiques différents. C'est pourquoi j'ai pu écrire sur Audiberti dans notre revue. D'autant qu'à mon avis il y a intérêt à multiplier les types d'appréhension des œuvres, sortir des habitudes, des langages établis. Puis critiquer les résultats impitoyablement.

31 - KRYNEN : Dans la convention qui nous rassemble, nous n'étudions pas le Baroque dans l'abstrait, mais une spécificité historiquement localisée, avec toutes les tensions qui constituent la crise de l'époque.

32 - Marguerite SOULIÉ : Même en restreignant plus encore l'objet, on trouve, par exemple dans la littérature protestante de la fin $\mathrm{du} \mathrm{XVI}^{\mathrm{e}}$ siècle, de considérables contradictions. C'est cela qui fait problème : les différences de fonctionnement.

33 - WAGNER : Mais, si parmi ces différences, il n'y avait des éléments communs, vous ne seriez pas tentée d'appliquer l'épithète baroque.

34 - CASTAN : Le problème posé, c'est le problème du contrôle: travail, résultats, démarches. Un mathématicien toulousain disait ici, au cours d'une précédente session, parlant de Fermat: les formalisations surviennent dans les moments de reflux. Lorsqu'une science est en pleine découverte, il n'existe pas de conceptualisation étroite. Mais que substituerons-nous à la conceptualisation? Le P. Costabel parle de classements structurants. Je crois en outre, avec F. Courtès et J-P. Martinon, qu'il faut s'interroger sur le lieu où se place le chercheur quand il travaille. Alors apparaît une notion sans laquelle il ne peut y avoir de science : la notion de laboratoire imaginaire. Le lieu, la communauté de recherche que nous constituons ensemble, et que nous avons pour objet en ce moment de toucher du doigt ou de repérer par la parole : cette communauté qui rassemble tous les chercheurs, et qui prend un caractère concret chaque fois qu'un congrès a lieu ou que paraît une revue. À partir de la notion de laboratoire imaginaire, une instance de contrôle s'instaure: alors les problèmes de périodisation ou de critère sont seconds. C'est le laboratoire dans son produit qui donne la vision et la réalité du Baroque. Tout ce qui prendra place dans la nébuleuse en formation fera partie du Baroque. Dans cette vue, il faut procéder à des expériences pour voir : voilà pourquoi nous avons un jour demandé à Auzias un article sur Audiberti. L'idée qui nous dirige cependant, c'est qu'il existe une période historique où se réalise l'idéal du Baroque. Autour de ce foyer, et parfois loin de lui, les formes peuvent trouver des correspondances, y compris à l'époque 
contemporaine. On utilise de même le terme de romantique pour caractériser éventuellement une œuvre qui n'appartient pas à la période où le Romantisme s'est élaboré, à grande échelle et dans sa complexité, comme un des idéaux possibles de l'esprit humain. L'important réside dans le laboratoire imaginaire. C'est le but et le programme. Il s'agit d'arracher le Baroque aux diverses disciplines, où il ne parvient pas à trouver l'autonomie de son lieu, un lieu de contrôle. Science en marge de l'Université telle qu'elle est aujourd'hui constituée. Défi. Fournir au Baroque les moyens d'un fonctionnement autonome.

35 - KRYNEN : Si les études baroques sont d'actualité, c'est vraisemblablement parce que nous sommes dans une période de crise dont les symptômes sont comparables à ceux de la période qui nous occupe.

36 - LAFAY : Depuis ce matin, on fait référence à un fait social global, auquel on parviendrait par accumulation de résultats partiels. N'est-ce pas trop optimiste? La recherche Spécifique ne tourne-t-elle pas plutôt le dos au fait de civilisation global? Dans mon domaine, je suis frappé par la non-littérarité du Baroque (au sens de Jacobson).

37 J'ai comme tout le monde rencontré le Baroque : si code il y a et grille de lecture, ils ont eu pour moi valeur heuristique. La présence mythique de ce code en moi est forte. Mais voici mon inquiétude: ma lecture de la poésie de cette période foisonnante était différente de celle qui ressortait de l'anthologie de Rousset, pourtant si riche. Ni les individualités ni l'ensemble de la production ne s'expliquaient par les critères énoncés. L'explication relevait parfois de critères contradictoires. Cela faisait donc problème. Je trouvais parfois les explications davantage dans des ouvrages d'historiens des mentalités (Mandrou) : on m'y parlait de désespoir, comme tout à l'heure le P. Costabel. Mais pourquoi appeler ce désespoir baroque ? J'étais là hors de mon système de repérage. Nous sommes dans un tunnel. Je pense que les études des spécialistes nous conduiront à renouveler la problématique. Je ne renonce pas.

38 - Discussion du rapport baroque/classique dans la production poétique : il va de soi que les effets d'équilibre par exemple peuvent avoir des significations toutes différentes selon les cas. On trouve chez Sponde des ostentations d'équilibre, chez Malherbe une projection de la rationalité de l'univers.

39 - GYENIS : Il n'y a pas un seul Baroque, mais plusieurs. Il y a aussi des phases dans le Baroque : naissance, maturité, formes tardives. Mandrou lui-même montre qu'au-dessous de la littérature des Lumières, y compris en France, vit une littérature pour le peuple, de caractère baroque. Comment comprendre qu'en Europe centrale, où la littérature reste baroque au XVIII ${ }^{\mathrm{e}}$ siècle, celle-ci puisse servir de véhicule aux Lumières?

40 - KRYNEN : Au début du XVII siècle surgit une théologie constitutive du Baroque, véritable théologie de la raison, des Lumières, par opposition à la théologie antérieure. Vigile d'une pensée laïque.

41 - On s'accorde pour noter la relation profonde de l'art populaire et de l'art baroque.

42 - Marguerite SOULIÉ : L'art de d'Aubigné se présente comme un art de la réalité, un art qui sert à vivre, par opposition à des codes figés. L'art populaire n'est donc pas loin. 


\section{NOTES}

1. Voir à ce propos les textes de Pierre Charpentrat: L'art Baroque, P.U.F., et, du même auteur, Baroque I, à l'office du Livre.

2. Voir à ce propos l'article de Paul Veyne : «Vie de Trimalcion », revue des Annales, Colin, 1961, $n^{\circ} 2$.

\section{AUTEUR}

\section{JEAN-CLAUDE MARTINON}

Maître-Assistant à l'U.E.R. Histoire-Sociologie Paris VIII - Membre du C.E.S.M. 